\title{
Implicaciones del formato en estilo editorial de revistas científicas indizadas mexicanas
}

\section{Implications of the format in editorial style of Mexican indexed journals}

\author{
Javier Tarango \\ ti.88888@hotmail.com \\ Profesor-Investigador Universidad Autónoma de Chihuahua (México) \\ Laura Patricia Murguía-Jáquez \\ pmurguia@,uach.mx \\ Profesor-Investigador Universidad Autónoma de Chihuahua (México) \\ Arturo Iván Ruiz-Domínguez \\ air ruiz2@msn.com \\ Director de la Biblioteca del Tribunal Supremo de Justicia del Estado de \\ Chihuahua (México), Profesor Universidad Autónoma de Chihuahua (México) \\ Fidel González-Quiñones \\ fgonzalez@uach.mx \\ Profesor-Investigador Universidad Autónoma de Chihuahua (México)
}

\section{Resumen}

La presente investigación (de carácter cuantitativo, no experimental, de finalidad exploratoria-descriptiva), estudia las problemáticas que observa la aplicación de diversos formatos de estilo editorial en la integración de artículos científicos y la complejidad en la migración de un formato a otro, debida a la amplia variedad de especificaciones que se demandan. A partir de la aplicación de un modelo conceptual simplificado (compuesto de 5 dimensiones y 17 criterios), se evaluaron los datos recurrentes de 204 revistas científicas mexicanas incluidas en el Sistema de Clasificación de Revistas Mexicanas de Ciencia y Tecnología del Consejo Nacional de Ciencia y Tecnología (CONACY'T), considerando: las características de la publicación y del documento, estructura de metadatos, identificación de manuales de estilo en el cuerpo del documento y clasificación de los estilos en la estructura de referencias. De acuerdo a la problemática planteada, se vislumbran diversas 
perspectivas: (i) búsqueda de la estandarización nacional en formatos de estilos editorial; (ii) integración de programas formales de instrucción y soporte académico para estudiantes, profesores e investigadores; y (iii) creación de perfiles profesionales en procesos de gestión en comunicación científica.

\title{
Palabras clave
}

Manuales de estilo; normas editoriales; formato editorial; artículos científicos; comunicación científica; producción científica; revistas arbitradas e indizadas; investigadores científicos; CONACYT; México.

\begin{abstract}
The present research (quantitative, non-experimental, exploratory-descriptive), studies the problems observed by the application of various formats of editorial style in the integration of scientific articles and the complexity in the migration from one format to another, because the wide variety of specifications that are demanded. Based on the application of a simplified conceptual model (composed of five dimensions and 17 criteria), the recurrent data of 204 scientific Mexican journals included in the Sistema de Clasificación de Revistas Mexicanas de Ciencia y Tecnología of the Consejo Nacional de Ciencia y Tecnología (CONACYT), considering: the characteristics of the publication and the document, structure of metadata, identification of style manuals in the body of the document and classification of styles in the structure of references. According to the problem posed, various perspectives are glimpsed: (i) search for national standardization in editorial style formats; (ii) integration of formal instructional and academic support programs for students, professors and researchers; and (iii) creation of professional profiles in management processes in scientific communication.
\end{abstract}

\section{Keywords}

Manuals of style; editorial standards; editorial format; scientific articles; scientific communication; scientific production; refereed and indexed journals; scientific researchers; CONACYT; Mexico. 
Recibido:29/11/2019

Aceptado:26/12/2019

DOI: https://dx.doi.org/10.5557/IIMEI10-N19-127158

Descripción propuesta: Tarango, Javier; Murguía-Jáquez, Laura Patricia; RuizDomínguez, Arturo Iván; González-Quiñones, Fidel, 2019. Implicaciones del formato en estilo editorial de revistas científicas indizadas mexicanas. Métodos de Información, 10(19), 127-158

\section{Introducción}

La integración de documentos para procesos de comunicación científica se compone de 2 elementos generales: el fondo y la forma. El primero, vinculado a los contenidos disciplinares que los investigadores desarrollan, preferentemente con enfoque disciplinar y con una visión hacia la creatividad y la innovación. El segundo, en relación al formato editorial, aunque de apariencia secundaria, representa un conjunto de criterios respecto a su estructura (distribución de contenidos, presencia de elementos editoriales básicos, tipografía, formas de citación y referenciación).

Respecto al fondo de los contenidos científicos, se espera que entre las competencias de los profesores e investigadores, la competencia de la identificación temática esté altamente desarrollada. En cambio, los aspectos relacionados con la forma se convierten en elementos obligatorios y varían en mayor medida dependiendo de cada revista científica en la que se desea contribuir, incluso se convierten en el primer filtro para determinar la continuación de procesos de evaluación para su publicación. La principal preocupación en este sentido, es cuando la forma de los documentos científicos toma mayor complejidad y preponderancia sobre el fondo o contenido de los mismos.

Poca evidencia se identifica en la literatura científica que critique la rigidez y variabilidad de los formatos de estilo, salvo la visión de Fierro (2004), quien considera que los manuales de estilo sólo cumplen con la función de inhibir la búsqueda de procesos de publicación. Contrario a esto, es frecuente encontrar gran variedad de documentos publicados que defienden la importancia de la 
sujeción a normatividades en la estructura de documentos científicos, como parte de la búsqueda de la uniformidad en la presentación de manuscritos (CONACYT, 2019a). Teóricamente, existe una propuesta generalizada a buscar alternativas de formación en procesos de gestión editorial y en la generación de normalizaciones funcionales relacionadas con disciplinas científicas particulares (Pei-Shan 2019).

Ante la preocupación en las dificultades del formato editorial, el objetivo general propuesto en esta investigación, es identificar la variabilidad en criterios de publicación de artículos científicos, estudiando las revistas incluidas en el Sistema de Clasificación de Revistas Mexicanas de Ciencia y Tecnología del Consejo Nacional de Ciencia y Tecnología (CONACYT, 2018), en un diseño transversal de datos vigentes a enero de 2019, con lo cual, fue posible identificar implicaciones en las dificultades para comunicar conocimiento por parte de profesores e investigadores que desean publicar en tales revistas científicas. La razón por la que se eligió este sistema de información como fuente para recolectar datos se debe a que el CONACYT, como órgano público descentralizado del gobierno federal mexicano, es la institución dedicada a promover el avance en investigación, innovación y modernización tecnológica, por lo que lo convierte en la autoridad científica del país.

\section{Condición de los profesores en su adaptación a los formatos de estilo}

El panorama de la comunicación científica adquiere una enorme complejidad si se atiende a los diversos canales y productos en los que se manifiesta (Peinado-Rodríguez 2013). Por tanto, se demanda que el investigador desarrolle capacidades de trabajo multifactorial, lo que provoca que se identifiquen mayores cualidades en algunos en comparación con otros (Sancho 2010; Prieto-Díaz et al. 2011). La tarea de los investigadores con más experiencia es bastante clara: formar a otros recursos humanos, aunque no siempre hay disposición a la enseñanza y el aprendizaje, tanto por quienes conocen como por quienes no (Pérez-Morales, Rodríguez-Manrique 2012). 
La adaptación de los profesores a los procesos de comunicación científica va a depender de 2 condiciones fundamentales (Fondón, Madero y Sarmiento 2010): (i) ser principiantes, pero mostrar amplia disposición a incorporarse en actividades relacionadas con la investigación; y (ii) aquellos con mayor número de años incorporados a las actividades académicas, que, no obstante, no poseen producción científica, así como tampoco valoran la posibilidad. Ambas situaciones son igualmente complejas cuando se trata de obtener resultados manifiestos a través de la cuantificación de su comunicación científica. En el caso de la primera situación, se esperaría que los profesores dediquen gran parte de su tiempo durante los primeros años a buscar formas de aprendizaje; en la segunda situación, los profesores buscan formas de adaptación a las exigencias propias de los indicadores de medición de la calidad de las universidades.

Otra situación que se presenta en los profesores que intentan desempeñar su labor dentro del ámbito de la investigación, es el desconocimiento de los marcos organizacionales para desenvolverse adecuadamente en distintas estructuras ajenas a su propia institución que, en este caso, son las agencias dedicadas a calificar las condiciones en sus procesos de comunicación científica (Vélez-Cuartas et al. 2014). Todo esto significa que mientras en la docencia la propia institución determina la calidad del profesor, en el caso de la comunicación científica, la decisión de la calidad del trabajo presentado estará resuelta por normas y reglas definidas de otros órganos de evaluación (Bornmann 2019).

Haciendo énfasis en la importancia del apoyo en la enseñanza en los procesos de investigación y comunicación científica, se considera que los procesos formativos no deben limitarse a meros aspectos conceptuales, procedimentales y axiológicos, intentado conseguir que se materialicen acciones concretas de alfabetización científica (Gavidia 2013), la cual debe ser concebida como un proceso orientado a la investigación, que permita a los sujetos participar en actividades científicas, enfrentarse a problemas relevantes y (re)construir los conocimientos científicos, que habitualmente la enseñanza trasmite basada en contenidos previamente elaborados, lo que favorece el aprendizaje eficiente y significativo (Vilches, Solbes y Gil 2004). 
En la actualidad, las universidades ponderan la comunicación científica como un elemento fundamental del desarrollo de profesores con miras a desempeñarse como investigadores, inmersos en los procesos de generación y aplicación innovadora de conocimiento. Sin embargo, de acuerdo a la realidad, los procesos de publicación ofrecen diversas restricciones (Pérez-Anaya 2017; Cordero-Arroyo et al. 2009): (i) existe carencia de programas formales para adquirir competencias científicas suficientes para generar textos científicos; (ii) los profesores suelen tener problemas para iniciar procesos de comunicación científica como una actividad cotidiana; (iii) dentro de las actividades de los profesores con alta carga de docencia, existen pocas oportunidades para desarrollar trabajo sistemático de investigación; y (iv) en los productos publicados suele valorarse la cantidad por encima de la calidad.

Aunque la comunicación científica ofrece múltiples variables, su principal actividad se refleja en el desarrollo y publicación de artículos científicos, que suman relevancia bajo los siguientes criterios (Asencio-Cabot, Ibarra-López 2018): (i) son algunos de los canales principales para la comunicación actual del conocimiento científico; (ii) representan la institucionalización social de la ciencia en la mayoría de los campos del conocimiento; (iii) generan procesos de indización que facilitan su visibilidad; (iv) los indicadores de las universidades actuales se basan principalmente en el número de artículos publicados en revistas científicas arbitradas e indizadas; y (v) es prácticamente el único tipo de documento en el que se mide el Factor de Impacto a través del número de citaciones.

En relación a la propuesta de este artículo, finalmente debe considerarse que tanto el fondo como la forma representan la arquitectura en la estructura de los artículos científicos y de cualquier documento, dando como consecuencia la posibilidad de que el investigador defina la integración de múltiples elementos de conocimiento, sin obviar reconocer distintas problemáticas asociadas a lograr la efectiva publicación de los hallazgos, como son (GangaContreras, Paredes-Buzeta y Pedraja-Rejas 2015; Alonso-Gamboa, Reyna Espinosa y Sánchez-Islas 2015): (i) las propias revistas científicas observan una compleja dinámica de trabajo; (ii) los tiempos de demora en la aceptación y publicación son prolongados; (iii) la cantidad limitada de artículos aceptados por emisión; y (iv) la desestimación de artículos para su publicación tomando 
como referencia elementos insignificantes relacionados regularmente con la forma y no con el fondo del contenido del documento.

Dado el peso que toma la forma de los manuscritos por encima del fondo de los contenidos, las cuestiones editoriales se han considerado que constituyen una actividad connatural de la actividad académica (López-Valdez 2009). Esta labor se estima crucial en el desarrollo de los procesos de calidad de la comunicación científica, representa la integración de obras especializadas y comprende aspectos legales, académicos, administrativos e incluso, comerciales. Se puede decir que es aquí donde la unificación de estilos y normas toma relevancia en la integración de documentos científicos. La defensa de la normalización en la integración de documentos científicos por su forma se justifica en las siguientes visiones: (i) tiene como fin tipificar, armonizar, estandarizar, homogeneizar, unificar y uniformar criterios para aplicarlos en una publicación que busca su propia identidad (Eguaras 2014); y (ii) es una guía interna de trabajo, destinada a orientar al propio autor y al personal editorial (Senz 2014).

Debido a que los procesos de comunicación científica, en suma son mayormente considerados como el fin último de los resultados en la medición de la competencia de las instituciones de educación superior, se vuelve necesario influir en la mejora de las deficiencias formales y metodológicas que experimentan los profesores bajo las siguientes condiciones (De Filippo, Marugán y Sanz-Casado 2014; Ortoll et al 2014; Rodríguez-Menéndez, González-Cantalapiedra y González-Polo 2016): (i) escasez en el desarrollo de mejores niveles de alfabetización científica; (ii) carencia de atención en el envío de artículos sin observar normas de estructuración y composición; (iii) pretender revelar contenidos sobreexplotados en las publicaciones ya existentes, así como plagio y autoplagio (voluntario o involuntario); y (iv) baja capacidad de trabajo en colaboración que limita la generación de conocimiento en dimensiones globales.

La perspectiva sobre la problemática que experimentan los profesores en la comunicación científica es una situación cultural. Aunque si esta barrera se supera, la siguiente situación corresponde a la capacidad en la preparación de manuscritos, los cuales van desde la creación de ideas innovadoras y la 
estructura armónica de los textos hasta la búsqueda de alternativas de publicación (Kratochvíl 2017).

\section{Metodología}

El carácter metodológico de este estudio ofrece un enfoque cuantitativo, de naturaleza investigativa no experimental, cuya finalidad es de tipo exploratoria y descriptiva, y atiende una temporalidad con un diseño transversal con un solo corte de tiempo. Su tipo de investigación es estratégica, ya que a partir de los resultados ofrece inferencias de propuestas concretas de acción ante un problema identificado.

Exprofeso se generó un modelo conceptual simplificado (véase Figura 1), a través del cual se identificaron 5 dimensiones de evaluación: (i) características de las publicaciones; (ii) características del documento; (iii) metadatos; (iv) manual de estilo en relación al formato del documento; y (v) manual de estilo en las referencias. De las 5 dimensiones se desagregaron 17 indicadores de medición. A partir del modelo propuesto se procedió a identificar las características específicas de cada revista participante en el estudio.

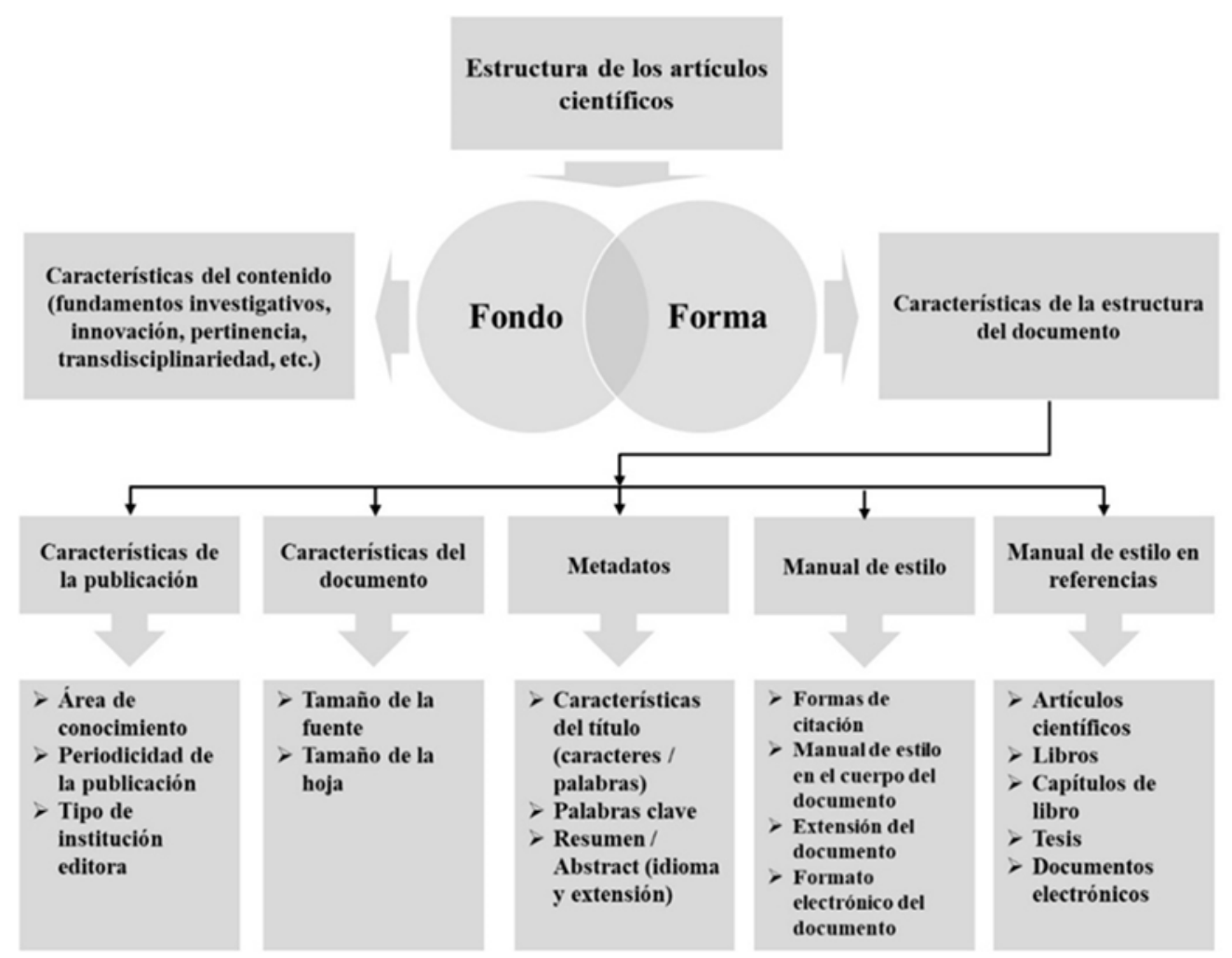

Figura 1. Modelo Conceptual Simplificado 


\section{Presentación y análisis de resultados}

\subsection{Características de la publicación}

Esta dimensión presenta un panorama nacional mexicano de revistas indizadas respecto a las fuentes destinadas a comunicar ciencia a través de los criterios siguientes:

- $\quad$ Periodicidad de las publicaciones. Este indicador observa 6 tipos de períodos de publicación: 5 de forma regular y otro irregular (véase Tabla 1). La frecuencia más común es la semestral con 84 publicaciones $(41.2 \%)$. Se estima que el número de fascículos (electrónicos o físicos) que se publican por año en México, a través del Sistema de Clasificación de Revistas Mexicanas de Ciencia y Tecnología del CONACYT, equivale a 631 con más de 5,000 artículos científicos.

\begin{tabular}{|c|c|c|c|}
\hline Periodicidad & Frecuencia & Porcentaje & $\begin{array}{l}\text { Porcentaje } \\
\text { acumulado }\end{array}$ \\
\hline Semestral & 84 & 41.20 & 41.20 \\
\hline Cuatrimestral & 47 & 23.00 & 64.20 \\
\hline Trimestral & 39 & 19.10 & 83.30 \\
\hline Bimestral & 26 & 12.70 & 96.00 \\
\hline Anual & 6 & 2.90 & 98.90 \\
\hline Otro & 2 & 1.10 & 100.00 \\
\hline Total & 204 & 100.00 & \\
\hline
\end{tabular}

Tabla 1. Periodicidad de las publicaciones

- Tipo de institución editora. Las instituciones generadoras y promotoras de conocimiento científico son determinantes en la definición de la calidad de las publicaciones, partiendo del criterio que observan la capacidad para lograrlo al contar de manera formal y sistemática con departamentos editoriales, cuerpos docentes y de investigación suficientes, redes epistémicas fortalecidas para acceso a árbitros experimentados, etc. Este criterio fue evaluado en 3 
categorías: por tipo de institución en sí, por el nombre de la institución editora y áreas de conocimiento.

Respecto al tipo de institución, se creó una clasificación arbitraria en 10 categorías. El $82.84 \%$ de las publicaciones provienen de 4 tipos de instituciones, siendo las universidades nacionales (clasificadas aquí las instituciones de administración federal como la Universidad Nacional Autónoma de México, Universidad Autónoma Metropolitana y el Instituto Politécnico Nacional) las de mayor aportación (36.76\%), seguido por asociaciones profesionales, universidades estatales (ubicadas en cada estado del país con carácter de autonomía) y centros de investigación (véase Tabla 2).

\begin{tabular}{llll}
\hline Tipo de institución & Frecuencia & Porcentaje & $\begin{array}{l}\text { Porcentaje } \\
\text { acumulado }\end{array}$ \\
\hline Universidad nacional & 75 & 36.76 & 36.76 \\
Asociación profesional & 39 & 19.12 & 55.88 \\
Universidad estatal & 35 & 17.16 & 73.03 \\
Centro de investigación & 20 & 9.80 & 82.84 \\
Centro de pensamiento & (Think & & \\
Tank) & 12 & 5.88 & 88.72 \\
Centro de salud pública & 12 & 5.88 & 94.60 \\
Universidad privada & 4 & 1.96 & 96.56 \\
Entidad gubernamental & 3 & 1.47 & 98.03 \\
Editorial & 2 & 0.98 & 99.01 \\
Empresa privada & 2 & 0.98 & 100.00 \\
\hline Total & $\mathbf{2 0 4}$ & $\mathbf{1 0 0 . 0 0}$ & \\
\hline
\end{tabular}

Tabla 2. Clasificación por tipo de institución

En relación a la institución editora se identificaron 125 entidades, de las cuales, 22 editan de 2 a 15 publicaciones y 103 sólo observan una frecuencia (véase Tabla 3).

\begin{tabular}{lccc}
\hline \multicolumn{1}{c}{ Institución editora } & Frecuencia & Porcentaje & $\begin{array}{c}\text { Porcentaje } \\
\text { acumulado }\end{array}$ \\
\hline UNAM $^{*}$ & 15 & 7.35 & 7.35 \\
Universidad Autónoma & 12 & 5.88 & 13.23
\end{tabular}




\begin{tabular}{|c|c|c|c|}
\hline Institución editora & Frecuencia & Porcentaje & $\begin{array}{l}\text { Porcentaje } \\
\text { acumulado }\end{array}$ \\
\hline \multicolumn{4}{|l|}{ Metropolitana } \\
\hline El Colegio de México, A.C. & 8 & 3.92 & 17.15 \\
\hline \multicolumn{4}{|l|}{ UNAM, Coordinación de } \\
\hline Humanidades & 8 & 3.92 & 21.08 \\
\hline Universidad de Guadalajara & 8 & 3.92 & 25.00 \\
\hline \multicolumn{4}{|l|}{ UNAM, Coordinación de la } \\
\hline Investigación Científica & 7 & 3.43 & 28.43 \\
\hline Instituto Politécnico Nacional & 5 & 2.45 & 30.88 \\
\hline \multicolumn{4}{|l|}{ UNAM, Instituto de Investigaciones } \\
\hline Jurídicas & 4 & 1.96 & 32.84 \\
\hline Universidad Autónoma Chapingo & 4 & 1.96 & 34.80 \\
\hline \multicolumn{4}{|l|}{ Universidad Autónoma de Baja } \\
\hline California & 4 & 1.96 & 36.76 \\
\hline Instituto de Ecología, A.C. & 3 & 1.47 & 38.23 \\
\hline \multicolumn{4}{|l|}{ Instituto Nacional de Investigaciones } \\
\hline Forestales, Agrícolas y Pecuarias & 3 & 1.47 & 39.70 \\
\hline \multicolumn{4}{|l|}{ Centro de Investigación y Docencia } \\
\hline Económicas, A.C. & 2 & 0.98 & 40.68 \\
\hline El Colegio de la Frontera Norte, A.C. & 2 & 0.98 & 41.66 \\
\hline \multicolumn{4}{|l|}{ Instituto de Investigaciones Dr. José } \\
\hline María Luis Mora & 2 & 0.98 & 42.64 \\
\hline \multicolumn{4}{|l|}{ Instituto Nacional de Antropología e } \\
\hline Historia & 2 & 0.98 & 43.62 \\
\hline \multicolumn{4}{|l|}{ UNAM, Instituto de Investigaciones } \\
\hline Históricas & 2 & 0.98 & 44.60 \\
\hline Universidad Autónoma de Chapingo* & 2 & 0.98 & 45.59 \\
\hline \multicolumn{4}{|l|}{ Universidad Autónoma de Chapingo, } \\
\hline Colegio de Postgraduados & 2 & 0.98 & 46.57 \\
\hline \multicolumn{4}{|l|}{ Universidad Autónoma de } \\
\hline Tamaulipas & 2 & 0.98 & 47.55 \\
\hline \multicolumn{4}{|l|}{ Universidad Autónoma del Estado de } \\
\hline México & 2 & 0.98 & 48.53 \\
\hline Universidad de Guanajuato & 2 & 0.98 & 49.51 \\
\hline Instituciones con una mención & 103 & 50.49 & 100.00 \\
\hline Total & 204 & 100.00 & \\
\hline
\end{tabular}

$\left(^{*}\right)$ No identifican dependencia específica.

Tabla 3. Listado por institución editora

- $\quad$ Área de conocimiento. Las revistas se agrupan en 8 áreas del conocimiento (según la propia clasificación del CONACYT a través 
del Sistema Nacional de Investigadores (SNI), 7 disciplinares y una multidisciplinaria (CONACYT, 2019b). Las ciencias sociales es el área con mayor aportación de publicaciones (37.3\%) (véase Tabla 4).

\begin{tabular}{|c|c|c|c|}
\hline Área & Frecuencia & $\%$ & $\begin{array}{c}\% \\
\text { acumulado }\end{array}$ \\
\hline Ciencias Sociales (Área V) & 76 & 37.30 & 37.30 \\
\hline Medicina y Ciencias de la Salud (Área III) & 35 & 17.20 & 54.50 \\
\hline $\begin{array}{l}\text { Humanidades y Ciencias de la Conducta } \\
\text { (Área IV) }\end{array}$ & 34 & 16.70 & 71.20 \\
\hline $\begin{array}{l}\text { Biotecnología y Ciencias Agropecuarias } \\
\text { (Área VI) }\end{array}$ & 19 & 9.30 & 80.50 \\
\hline $\begin{array}{l}\text { Físico-Matemáticas y Ciencias de la Tierra } \\
\text { (Área I) }\end{array}$ & 13 & 6.40 & 86.90 \\
\hline Ingenierías (Área VII) & 10 & 4.90 & 91.80 \\
\hline $\begin{array}{l}\text { Biología, Química y Ciencias de la Vida } \\
\text { (Área II) }\end{array}$ & 10 & 4.80 & 96.60 \\
\hline Multidisciplinarias (Área VIII) & 7 & 3.40 & 100.00 \\
\hline Total & 204 & 100.00 & \\
\hline
\end{tabular}

Tabla 4. Distribución de revistas por área de conocimiento

\subsection{Características del documento}

Se estudian 2 tipos: tamaño de la fuente (tipografía) y tamaño de la hoja (cuartilla). Estos criterios pueden resultar los criterios más sencillos dada su posibilidad de migración en caso de cambio de una publicación a otra.

- $\quad$ Tamaño de la fuente. Se identifican 4 opciones: 12 puntos $(75.50 \%)$; no especificado (18.10\%); 11 puntos (3.40\%); y 10 puntos (2.91\%).

- Tamaño de la hoja. El $75.50 \%$ no especifica y el $24.50 \%$ indica que tamaño carta.

\subsection{Metadatos}

Son considerados fundamentales en la visibilidad de contenidos científicos. En este caso se incluyen los siguientes elementos de 
evaluación: características del título, palabras clave/Keywords y resumen/Abstract.

- Características del título. Este criterio se caracteriza por 5 rasgos: no especifica, palabras, caracteres, breve y líneas. Su mayor frecuencia se centra en la ausencia de especificaciones (55.88\%). Las formas tangibles de medición son representadas en número de palabras, caracteres y líneas. Algunas revistas científicas caracterizan a los títulos bajo el rasgo genérico de "breve" (véase Tabla 5).

\begin{tabular}{lccc}
\hline Características & Frecuencia & Porcentaje & $\begin{array}{c}\text { Porcentaje } \\
\text { acumulado }\end{array}$ \\
\hline No especifica & 114 & 55.88 & 55.88 \\
Palabras & 45 & 22.06 & 77.94 \\
Caracteres & 27 & 13.24 & 91.17 \\
Breve & 16 & 7.84 & 99.02 \\
Líneas & 2 & 0.98 & 100.00 \\
\hline \multicolumn{1}{c}{ Total } & $\mathbf{2 0 4}$ & $\mathbf{1 0 0 . 0 0}$ & \\
\hline
\end{tabular}

Tabla 5. Características del titulo

- Palabras clave/Keywords. Este criterio observó múltiples variables en sus formas posibles de presentación, caracterizado por lo siguiente: (i) definición concreta de cantidad de palabras clave; (ii) rangos en máximos y/o mínimos; (iii) especificaciones de cantidad de palabras clave, fuentes de consulta o restricciones en su asignación; y (iv) no especifica (véase Tabla 6).

\begin{tabular}{cccc}
\hline Palabras clave & Frecuencia & Porcentaje & $\begin{array}{c}\text { Porcentaje } \\
\text { acumulado }\end{array}$ \\
\hline 5 & 61 & 29.90 & 29.9 \\
No especifica & 38 & 18.63 & 48.53 \\
$3-5$ & 24 & 11.76 & 60.29 \\
$4-6$ & 11 & 5.39 & 65.68 \\
$3-6$ & 9 & 4.41 & 70.10
\end{tabular}




\begin{tabular}{|c|c|c|c|}
\hline Palabras clave & Frecuencia & Porcentaje & $\begin{array}{l}\text { Porcentaje } \\
\text { acumulado }\end{array}$ \\
\hline $3-10$ & 7 & 3.43 & 73.53 \\
\hline 6 & 7 & 3.43 & 76.96 \\
\hline $4-5$ & 6 & 2.94 & 79.90 \\
\hline 3 & 4 & 1.96 & 81.86 \\
\hline $5-6$ & 4 & 1.96 & 83.82 \\
\hline $5-10$ & 3 & 1.47 & 85.29 \\
\hline $5-7$ & 3 & 1.47 & 86.76 \\
\hline 8 & 3 & 1.47 & 88.23 \\
\hline 5 & 2 & 0.98 & 89.21 \\
\hline 6 & 2 & 0.98 & 90.19 \\
\hline 5 máximo & 2 & 0.98 & 91.17 \\
\hline 6 máximo & 3 & 1.47 & 92.65 \\
\hline 10 & 1 & 0.49 & 93.14 \\
\hline $1-5$ & 1 & 0.49 & 93.63 \\
\hline $1-6$ & 1 & 0.49 & 94.12 \\
\hline $2-5$ & 1 & 0.49 & 94.61 \\
\hline 3 mínimo & 1 & 0.49 & 95.10 \\
\hline $3-4$ & 1 & 0.49 & 95.59 \\
\hline $3-7$ & 1 & 0.49 & 96.08 \\
\hline 4 & 1 & 0.49 & 96.57 \\
\hline $4-40$ & 1 & 0.49 & 97.06 \\
\hline $4-8$ & 1 & 0.49 & 97.55 \\
\hline $\begin{array}{c}5 \text { máximo (en } \\
\text { clasificación JEL) }\end{array}$ & 1 & 0.49 & 98.04 \\
\hline 5 (preferentemente 3 & & & \\
\hline temáticas y 2 geográficas) & 1 & 0.49 & 98.53 \\
\hline $5-8$ & 1 & 0.49 & 99.02 \\
\hline $6-8$ & 1 & 0.49 & 99.51 \\
\hline 7 & 1 & 0.49 & 100.00 \\
\hline Total & 204 & 100.00 & \\
\hline
\end{tabular}

Tabla 6. Especificaciones de palabras clave

- Resumen/Abstract. Son evaluados desde 2 perspectivas: idioma en que se solicita y extensión en palabras. Respecto al idioma, se identificaron 8 alternativas probables (véase Tabla 7) que se caracterizan por el uso de 1, 2 y 3 idiomas y que un gran porcentaje de revistas no especifican. 


\begin{tabular}{|c|c|c|c|}
\hline Idioma del resumen & Frecuencia & Porcentaje & $\begin{array}{l}\text { Porcentaje } \\
\text { acumulado }\end{array}$ \\
\hline Inglés y español & 112 & 54.90 & 54.90 \\
\hline No especifica & 80 & 39.22 & 94.12 \\
\hline $\begin{array}{l}\text { Francés, inglés y } \\
\text { español }\end{array}$ & 5 & 2.45 & 96.57 \\
\hline Idioma del documento & 3 & 1.47 & 98.04 \\
\hline Español & 1 & 0.49 & 98.53 \\
\hline Inglés & 1 & 0.49 & 99.02 \\
\hline $\begin{array}{l}\text { Inglés, portugués y } \\
\text { francés }\end{array}$ & 1 & 0.49 & 99.51 \\
\hline Lengua original e inglés & 1 & 0.49 & 100.00 \\
\hline Total & 204 & 100.00 & \\
\hline
\end{tabular}

Tabla 7. Especificaciones de idioma del resumen

En relación con la extensión del resumen se distinguen 24 opciones agrupadas como sigue: (i) cantidad de palabras (mínimos y máximos); (ii) no especifica; e (iii) indicaciones vagas e imprecisas como: amplio o proporcional (Tabla 8).

\begin{tabular}{cccc}
\hline Extensión del resumen* & Frecuencia & & $\begin{array}{c}\text { Porcentaje } \\
\text { Porcentaje }\end{array}$ \\
acumulado
\end{tabular}




\begin{tabular}{|c|c|c|c|}
\hline Extensión del resumen* & Frecuencia & Porcentaje & $\begin{array}{l}\text { Porcentaje } \\
\text { acumulado }\end{array}$ \\
\hline 110 & 1 & 0.49 & 89.71 \\
\hline 160 & 1 & 0.49 & 90.20 \\
\hline 175 & 1 & 0.49 & 90.69 \\
\hline 251 & 1 & 0.49 & 91.18 \\
\hline 260 & 1 & 0.49 & 91.67 \\
\hline 350 & 1 & 0.49 & 92.16 \\
\hline 450 & 1 & 0.49 & 92.65 \\
\hline 1,500 caracteres contando espacios & 1 & 0.49 & 93.14 \\
\hline $100-150$ & 1 & 0.49 & 93.63 \\
\hline $100-175$ & 1 & 0.49 & 94.12 \\
\hline $100-200$ & 1 & 0.49 & 94.61 \\
\hline $100-300$ & 1 & 0.49 & 95.10 \\
\hline $120-150$ & 1 & 0.49 & 95.59 \\
\hline 15-20 líneas & 1 & 0.49 & 96.08 \\
\hline 500 caracteres & 1 & 0.49 & 96.57 \\
\hline 500 & 1 & 0.49 & 97.06 \\
\hline 8 a 10 líneas & 1 & 0.49 & 97.55 \\
\hline 850 caracteres & 1 & 0.49 & 98.04 \\
\hline 9 a 10 líneas & 1 & 0.49 & 98.53 \\
\hline Contenido amplio & 1 & 0.49 & 99.02 \\
\hline Contenido proporcional & 1 & 0.49 & 99.51 \\
\hline Una cuartilla & 1 & 0.49 & 100.00 \\
\hline Total & 204 & 100.00 & \\
\hline
\end{tabular}

(*) Las cifras expresadas sin alguna indicación representan cantidad de palabras.

\section{Tabla 8. Especificaciones de la extensión de resumen}

\subsection{Características del documento}

Este criterio regularmente se refiere a la totalidad de la forma como se presenta el documento, pero para efectos de este apartado se analizan las opciones que solicitan las revistas en evaluación en 3 aspectos fundamentales del documento: manual de estilo en el cuerpo del documento, forma de citación en el texto y extensión del documento.

- Manual de estilo en el cuerpo del documento. Se identificaron 15 variantes, aunque 163 de 204 revistas evaluadas (79.90\%) usan un 
manual propio, lo cual implica una variedad vasta de alternativas basadas en requerimientos individuales. Otras opciones características son: (i) especificación precisa de un manual estandarizado; y (ii) combinación de manuales, regularmente indicando alguno para el cuerpo del documento y otro para el registro de referencias (véase Tabla 9).

\begin{tabular}{lccc}
\hline $\begin{array}{c}\text { Manual de estilo en el cuerpo del } \\
\text { documento }\end{array}$ & Frecuencia & Porcentaje & $\begin{array}{c}\text { Porcentaje } \\
\text { acumulado }\end{array}$ \\
\hline $\begin{array}{l}\text { Manual propio } \\
\text { APA }\end{array}$ & 163 & 79.90 & 79.90 \\
Manual propio, Referencias en & 17 & 8.33 & 88.23 \\
Formato APA & 7 & 3.43 & 91.66 \\
$\begin{array}{l}\text { Estilo Harvard } \\
\text { LaTeX }\end{array}$ & 4 & 1.96 & 93.63 \\
Manual de Estilo Chicago & 2 & 0.98 & 94.61 \\
Comité Internacional de Editores de & 2 & 0.98 & 95.59 \\
Revista Médicas & 1 & & \\
Formato Bluebook & 1 & 0.49 & 96.08 \\
Guía de estilo de Estudios de Asia y & & & 96.57 \\
Africa & 1 & 0.49 & 97.06 \\
IEEE & 1 & 0.49 & 97.55 \\
Manual propio y referencias en & & & \\
manual de estilo Index Medicus & 1 & 0.49 & 98.04 \\
Manual propio y referencias en APA & & & \\
O Vancouver & 1 & 0.49 & 98.53 \\
MLA & 1 & 0.49 & 99.02 \\
Physical Review Style & 1 & 0.49 & 99.51 \\
Vancouver & 1 & 0.49 & 100.00 \\
\hline \multicolumn{2}{r}{ Total } & $\mathbf{1 0 0 . 0 0}$ & \\
\hline
\end{tabular}

Tabla 9. Especificaciones de manual de estilo en cuerpo del documento

- Formas de citación. Se reconocen 19 opciones, siendo el dato crítico el alto porcentaje de frecuencias en la opción de "no especifica" (30.88\%). La variabilidad en la especificación de criterios de formas de citación entre cada opción regularmente está diferenciada por detalles mínimos, tales como: signos de puntuación, uso de mayúsculas/minúsculas, etc. (véase Tabla 10). 


\begin{tabular}{lccc}
\hline \multicolumn{1}{c}{ Formas de citación } & Frecuencia & Porcentaje & $\begin{array}{c}\text { Porcentaje } \\
\text { acumulado }\end{array}$ \\
\hline No especifica & 63 & 30.88 & 30.88 \\
(Apellido, año) o Apellido (año) & 56 & 27.45 & 58.33 \\
(Apellido, año: página) & 31 & 15.20 & 73.53 \\
Apellido (año) o (Apellido, año) & 29 & 14.22 & 87.74 \\
(Apellido, año, página) & 11 & 5.39 & 93.13 \\
(Apellido año, página) & 4 & 1.96 & 95.10 \\
Notas al pie de página & 2 & 0.98 & 96.08 \\
(Apellido, año, p. XX) & 2 & 0.98 & 97.06 \\
(Apellido Año) & 1 & 0.49 & 97.55 \\
(Apellido Iniciales del nombre, año) & 1 & 0.49 & 98.04 \\
(Apellido Iniciales del nombre, año, & & & \\
página). & 1 & 0.49 & 98.53 \\
(Apellido: Página) & 1 & 0.49 & 99.02 \\
Apellido año: página & 1 & 0.49 & 99.51 \\
Numeradas con superíndice & 1 & 0.49 & 100.00 \\
\hline \multicolumn{1}{c}{ Total } & $\mathbf{2 0 4}$ & $\mathbf{1 0 0 . 0 0}$ & \\
\hline
\end{tabular}

Tabla 10. Especificaciones de formas de citación

- Extensión del documento. Se expresa de 2 formas: (i) conteo por cuartillas (56.4\%) que oscilan entre una extensión de 7 a 40 cuartillas; y conteo por palabras $(24 \%)$ con un margen de 3,500 a 35,000. El 19.7\% de las revistas no especifican requerimientos de extensión del documento.

- Formato electrónico del documento. Los formatos electrónicos solicitados son: Word (47.5\%); no especificado (43.6\%); recurso abierto o Word (4.9\%); PDF o Word (2.5\%); y PDF (1.5\%).

\subsection{Manual de estilo en referencias}

En este criterio de evaluación, primero se listan los manuales de estilo demandados para referenciación de documentos y posteriormente, se registran las propuestas de referencia de: artículos científicos, libros, capítulos de libro, tesis y documentos electrónicos.

- Tipo de manual de estilo en referencias. La identificación de los nombres de los manuales de estilo demandados indica que el $45.59 \%$ de 
las revistas solicitan la aplicación de un manual propio. El resto de las revistas demandan el uso de 14 tipos más, siendo APA el de mayor frecuencia (véase Tabla 11).

\begin{tabular}{|c|c|c|c|}
\hline Manual de estilo en referencias & Frecuencia & Porcentaje & $\begin{array}{l}\text { Porcentaje } \\
\text { acumulado }\end{array}$ \\
\hline Manual propio & 93 & 45.59 & 45.59 \\
\hline APA & 53 & 25.98 & 71.57 \\
\hline Citing Medicine (2a Edición) de The & & & \\
\hline National Library of Medicine (NLM) & 18 & 8.82 & 80.39 \\
\hline Estilo Harvard & 9 & 4.41 & 84.80 \\
\hline No especifica & 7 & 3.43 & 88.23 \\
\hline Manual de Estilo Chicago & 6 & 2.94 & 91.17 \\
\hline Vancouver & 5 & 2.45 & 93.62 \\
\hline MLA & 4 & 1.97 & 95.59 \\
\hline LaTeX & 2 & 0.98 & 96.57 \\
\hline ACS citation guide & 1 & 0.49 & 97.06 \\
\hline Formato Bluebook & 1 & 0.49 & 97.55 \\
\hline $\begin{array}{l}\text { Guía de estilo de Estudios de Asia y } \\
\text { África }\end{array}$ & 1 & 0.49 & 98.04 \\
\hline IEEE & 1 & 0.49 & 98.53 \\
\hline ISO (Norma 690) & 1 & 0.49 & 99.02 \\
\hline Manual propio y referencias en APA & 1 & 0.49 & 99.51 \\
\hline Physical Review Style & 1 & 0.49 & 100.00 \\
\hline Total & 204 & 100.00 & \\
\hline
\end{tabular}

Tabla 11. Especificaciones de manual de estilo en referencias

- Formas de referenciación de artículos científicos. Se distinguieron 89 propuestas de referenciación de artículos científicos. El 16.18\% de las revistas en evaluación no especifican formato. 80 revistas $(39.22 \%)$ registran sólo una frecuencia (véase Tabla 12).

\section{Artículos científicos}

Frecuencia Porcentaje

Apellido (s), N. N. \& Apellido (s), N. N.

(Mes (es), año). Título del artículo.

Nombre de la revista,

Volumen(Número), páginas.
46

33
22.55

22.55

No especifica

$16.18 \quad 38.73$




\section{Artículos científicos \\ Frecuencia Porcentaje \\ Porcentaje \\ acumulado}

Apellido (s) NN, Apellido (s) NN,

Apellidos, NN. Título del artículo.

Nombre de la revista abreviado. Año;

número: páginas.

17

8.33

47.06

Apellido (s), N. N., Apellido (s), N. N.,

Apellido (s), N. N. (Año) "Título del

artículo", Nombre de la revista,

Volumen (Número), pp. XX-XX.

7

50.49

Nombre completo Apellidos, "Título

del artículo", Nombre de la revista

Número (mes(es) de Año): páginas.

$\begin{array}{lll}5 & 2.45 & 52.94\end{array}$

Apellido (s) NN, Apellido (s) NN,

Apellido (s) NN. Título del artículo.

Nombre de la revista Año;

volumen(número): páginas.

4

1.96

54.90

Apellido (s), Nombre completo. "Título

del artículo", en Nombre de la revista,

volumen: número (año): XX-XX.

$\begin{array}{lll}4 & 1.96 & 56.86\end{array}$

Apellido (s) NN, Apellido (s) NN,

Apellido (s) NN, Apellidos NN,

Apellidos NN. Título del artículo.

Nombre de la revista abreviado Año;

número o volumen: páginas.

3

1.47

58.33

Apellido (s), Nombre completo y

Nombre completo Apellido (s). Año.

Título del artículo, País. Nombre de la

revista, Volumen(Número), mes (es):

páginas.

1.47

59.80

Apellido (s), Nombre completo (Año).

Título del artículo. Nombre de la

\begin{tabular}{cccc} 
revista, Número, páginas. & 2 & 0.98 & 60.79 \\
Otros particulares con una mención & 80 & 39.22 & 100.00 \\
\hline Total & $\mathbf{2 0 4}$ & $\mathbf{1 0 0 . 0 0}$ & \\
\hline
\end{tabular}

Tabla 12. Especificaciones de referenciación de artículos científicos

- Formas de referenciación de libros. Los resultados muestran 82 opciones de referenciación de libros. 9 estilos muestran de 2 a 47 frecuencias. El 18.47\% no especifica estilo y 72 revistas científicas registran sólo una frecuencia (35.29\%) (véase Tabla 13). 


\section{Libros}

Frecuencia Porcentaje

Porcentaje acumulado

Apellidos (s), N. N., Apellido (s), N.

N. \& Apellido (s), N. N. (Año).

Título del libro (Edición). Lugar de

edición: Editorial.

47

23.04

23.04

No especifica

37

18.14

41.18

Apellidos (s) NN, Apellido (s), NN, Apellido (s), NN. Título del libro.

Edición. Lugar de edición. Editorial.

Año.

17

8.33

49.51

Apellidos (s), N. N., Apellido (s), N.

N., Apellido (s), N. N. (Año). Título

del libro (Edición). Lugar de edición:

Editorial.

12

5.88

55.39

Nombre completo Apellido (s) y

Nombre completo Apellido

(s), Título del libro (Lugar de edición:

Editorial, Año), páginas.

5

2.45

57.84

Apellido (s), Nombre completo,

Apellido (s), Nombre completo,

Apellidos (s), Nombre completo.

Título del libro. Lugar de edición:

Editorial, Año.

4

Apellido (s) NN, Apellido (s) NN,

Apellido (s) NN. Título del libro.

Edición. Lugar de edición: Editorial,

Año; XXX-XXX.

2

0.98

60.79

Apellido (s) NN, Apellido (s) NN,

Apellido (s) NN. Título del libro.

Edición. Lugar de edición: Editorial;

Año.

2

0.98

61.77

Apellido (s), N.N., Apellido (s), N.N., Apellido (s). (Año). Título del libro.

Lugar de edición: Editorial. 2

Apellido (s), Nombre completo y

Nombre completo Apellido (s). Año.

Título del libro. Trad. Nombre

completo Apellido (s). Editorial.

(Colección o serie). País.

2

0.98

63.73

Apellido (s), Nombre completo, Apellido (s), Nombre completo, Apellido (s), Nombre completo (Año). Título (Edición). Lugar de

2

0.98

64.71 


\begin{tabular}{cccc}
\hline Libros & Frecuencia & Porcentaje & $\begin{array}{c}\text { Porcentaje } \\
\text { acumulado }\end{array}$ \\
\hline edición: Editorial. & & & \\
Otros particulares con una mención & 72 & 35.29 & 100.00 \\
\hline Total & $\mathbf{2 0 4}$ & $\mathbf{1 0 0 . 0 0}$ & \\
\hline
\end{tabular}

Tabla 13. Especificaciones de referencias de libros

- Formas de referenciación de capítulos de libro. Se detallaron 77 variables de tipos de referencias de capítulos de libro. 54 revistas $(17.16 \%)$ no especifican formato. 67 revistas $(32.84 \%)$ registran sólo una frecuencia (véase Tabla 14).

\begin{tabular}{lccc}
\hline \multicolumn{1}{c}{ Capítulos de libro } & Frecuencia & Porcentaje & $\begin{array}{c}\text { Porcentaje } \\
\text { acumulado }\end{array}$ \\
\hline No especifica & 54 & 17.16 & 43.63 \\
Apellido (s), N. N., Apellido (s), N. & & & \\
N. \& Apellido (s), N. N. (Año). & & & \\
Título del capítulo. En: Apellido (s), & & \\
N. N., Apellido (s), N. N. \& & & \\
Apellido (s), N. N. Título del libro & & \\
(pp. XX-XX). Lugar de edición: & & \\
Editorial. & & \\
Apellido (s), NN., Apellido (s) NN., & & \\
Apellido (s), NN. Título del & & \\
capítulo. En: Apellido (s), NN, & & \\
Apellido (s) NN, ed. Título del & & \\
libro. Edición. Lugar de edición. & & \\
Editorial, Año. XX-XX. & & \\
Apellido (s), N. N., Apellido (s), N. & & \\
N., Apellido (s), N. N. (Año) & & \\
"Título del capítulo". En: Apellido & & & \\
(s), N. N. et al. Eds. Título del libro. & & & \\
Lugar de edición: Libro. & & \\
[Número consecutivo]. N. N. & & \\
Apellido (s), N. N. Apellido (s), N. & & \\
N. Apellido (s). Título del capítulo, & & \\
in: Título del libro, Ed. N. N. \\
Apellido (s) (Editorial, Año) p. XX- \\
XX.
\end{tabular}


Capítulos de libro

Frecuencia Porcentaje

Porcentaje acumulado

Número consecutivo en superíndice

Nombre completo Apellidos,

"Título del capítulo", en Título del

libro, coord. Nombre completo

Apellido (s) (Lugar de publicación:

Editorial - Editorial - Editorial,

Año), XX-XX.

6

2.94

61.27

Apellido, Nombre completo.

"Título del capítulo", en Título del

Libro. Nombre completo Apellido

(s) y Nombre completo Apellido (s)

(función abreviada). Lugar:

Editorial, Año: XX-XX del capítulo.

4

1.96

63.23

Apellido (s) NN, Apellido (s) NN,

Apellido (s) NN. Título del artículo.

En: Apellido (s) NN, Apellido (s)

NN, Apellido (s) NN, eds. Título

del libro. Edición. Lugar de edición:

Editorial; Año. pp. XXX-XXX.

2

0.98

64.22

Apellido (s), N. N., Apellido (s), N.

N. \& Apellido (s), N. N. Año.

Título del capítulo. Pp. XXX-XXX.

In: N. N. Apellido (s), N. N.

Apellido (s) \& N. N. Apellido (s)

(Eds.). Título del libro. Editorial,

Lugar de edición.

2

0.98

65.20

Apellido (s), Nombre completo y

Nombre completo Apellido (s).

Año. Título del capítulo, en Título

del libro (ed.), Editorial (Colección

o serie Número). País: XX-XX.

2

0.98

66.18

Apellido (s), Nombre completo, Apellido (s), Nombre completo, Apellido (s), Nombre completo (Año). Título del capítulo. En N. N.

Apellido (s) y N. N. Apellido (s)

(Eds.), Título del libro (pp. XX-

$\mathrm{XX}$ ). Lugar de publicación:

Editorial.

Otros particulares con una mención

\section{Total}

67

0.98

67.16

Tabla 14. Especificaciones de referencias de capitulos de libro 
- Tesis. Comprende 46 opciones de referenciación de tesis. 105 revistas (51.47\%) no especifican indicaciones y 4 revistas (1.94\%) prohíben incluir este tipo de documentos como referencias y 40 revistas científicas (19.61\%) registran sólo una frecuencia (véase Tabla 15).

\begin{tabular}{|c|c|c|c|}
\hline Tesis & Frecuencia & Porcentaje & $\begin{array}{l}\text { Porcentaje } \\
\text { acumulado }\end{array}$ \\
\hline No especifica & 105 & 51.47 & 51.47 \\
\hline $\begin{array}{l}\text { Apellido (s), N. N. (Año). Título } \\
\text { de la tesis (Tesis de XXX) } \\
\text { Institución donde se obtuvo el } \\
\text { grado, País. }\end{array}$ & 32 & 15.69 & 67.16 \\
\hline $\begin{array}{l}\text { Apellido (s), N. N. (Año) Título de } \\
\text { la tesis. Nivel o grado académico. } \\
\text { Institución que otorga el grado. }\end{array}$ & 8 & 3.92 & 71.08 \\
\hline $\begin{array}{l}\text { [Número consecutivo]. N. N. } \\
\text { Apellido (s), Tesis de XXX “Título } \\
\text { de la tesis” (Institución donde se } \\
\text { obtuvo el grado, Año). } \\
\text { http:/ /www.XXXXX }\end{array}$ & 6 & 2.94 & 74.02 \\
\hline $\begin{array}{l}\text { Nombre Apellido (s), “Título de la } \\
\text { tesis" (Tesis de XXX, Lugar de } \\
\text { publicación, Institución en donde } \\
\text { se obtuvo el grado, año), páginas. }\end{array}$ & 5 & 2.45 & 76.47 \\
\hline Prohibido citar tesis o no aplica & 4 & 1.96 & 78.43 \\
\hline $\begin{array}{l}\text { Apellido (s), N. N., Año, Título de } \\
\text { la tesis: Lugar de publicación, país: } \\
\text { Institución donde se obtuvo el } \\
\text { grado, tesis de XXX, XX pp. }\end{array}$ & 2 & 0.98 & 79.41 \\
\hline $\begin{array}{l}\text { Apellido (s), Nombre completo y } \\
\text { Nombre completo Apellido (s). } \\
\text { Año. Título de la tesis, tesis de } \\
\text { XXX. Departamento-Facultad- } \\
\text { Universidad. Ciudad. }\end{array}$ & 2 & 0.98 & 80.39 \\
\hline $\begin{array}{l}\text { Otros particulares con una } \\
\text { mención }\end{array}$ & 40 & 19.61 & 100.00 \\
\hline Total & 204 & 100.0 & \\
\hline
\end{tabular}

Tabla 15. Especificaciones de referencias de tesis

- Documentos electrónicos. Este tipo de documentos ofrece amplia variabilidad, por lo que en este estudio se consideraron sólo aquellos 
tipos que indican una liga electrónica para su localización (HTTP). En este criterio coinciden 75 opciones de referenciación, donde 76 revistas científicas $(37.25 \%)$ no especifican instrucciones de referenciación de este tipo de documentos. 67 revistas científicas evaluadas (32.84\%) registran sólo una frecuencia (véase Tabla 16).

\begin{tabular}{|c|c|c|c|}
\hline Documentos electrónicos & Frecuencia & Porcentaje & $\begin{array}{l}\text { Porcentaje } \\
\text { acumulado }\end{array}$ \\
\hline No especifica & 76 & 37.25 & 37.25 \\
\hline $\begin{array}{l}\text { Autor corporativo. Título del } \\
\text { documento. (Año). Recuperado de } \\
\text { http:/ /www.XXXXX. }\end{array}$ & 34 & 16.67 & 63.92 \\
\hline $\begin{array}{l}\text { Apellido(s), NN (año) Título de la } \\
\text { página web. Disponible en: URL } \\
\text { [Consultado día-mes-año] }\end{array}$ & 7 & 3.43 & 57.35 \\
\hline $\begin{array}{l}\text { [Número consecutivo]. Título del } \\
\text { documento (Editorial, Lugar de } \\
\text { publicación, Año). } \\
\text { http://www.XXXXX. }\end{array}$ & 5 & 2.45 & 59.80 \\
\hline $\begin{array}{l}\text { Nombre completo Apellido (s), } \\
\text { "Título del } \\
\text { documento", Fuente, http://XXXX } \\
\text { X (consultado el Día de Mes de } \\
\text { Año). }\end{array}$ & 5 & 2.45 & 62.25 \\
\hline $\begin{array}{l}\text { Apellido (s), Nombre (s). Título. } \\
\text { Ensayo en línea disponible en } \\
<\text { http://www.ciudadseva.com/text } \\
\text { os/.htm> [fecha de consulta: dia de } \\
\text { mes de año]. }\end{array}$ & 4 & 1.96 & 64.21 \\
\hline $\begin{array}{l}\text { Apellido (s), N. N., Año, Título del } \\
\text { documento (en línea): Lugar, } \\
\text { Institución, actualización: Día de } \\
\text { Mes de Año, } \\
<\text { http://www.XXXXX>, consulta: } \\
\text { Día de Mes de Año }\end{array}$ & 2 & 0.98 & 65.19 \\
\hline $\begin{array}{l}\text { Apellido (s), Nombre completo y } \\
\text { Nombre completo Apellido (s). } \\
\text { Año. Título del documento. Título } \\
\text { de la página Web o de la fuente. } \\
<\text { http://www.XXXXX>. }\end{array}$ & & & \\
\hline Consultado el Día de Mes de Año. & 2 & 0.98 & 66.17 \\
\hline $\begin{array}{l}\text { Autor corporativo. (Año). Título del } \\
\text { documento. Lugar de }\end{array}$ & 2 & 0.98 & 67.15 \\
\hline
\end{tabular}




\begin{tabular}{cccc}
\hline \multicolumn{1}{c}{ Documentos electrónicos } & Frecuencia & Porcentaje & $\begin{array}{c}\text { Porcentaje } \\
\text { acumulado }\end{array}$ \\
\hline $\begin{array}{l}\text { edición: Editorial. Recuperado de } \\
\text { http://www.XXXXX }\end{array}$ & & & \\
Otros particulares con una mención & 67 & 32.84 & 100.00 \\
\hline Total & $\mathbf{2 0 4}$ & $\mathbf{1 0 0 . 0 0}$ & \\
\hline
\end{tabular}

Tabla 16. Especificaciones de referencias de documentos electrónicos

\section{Conclusión y discusiones}

En el caso de esta investigación, el análisis de los datos recolectados, permitió mensurar la complejidad de la situación que observan las revistas científicas mexicanas en cuanto a sus demandas de elementos de formato. Los aspectos evaluados que representaron mayor complejidad son: (i) el exceso de opciones de manuales de estilo propios de cada revista científica; (ii) las desmesuradas maneras de registro de referencias en los 5 tipos de documentos estudiados, observándose diferencias mínimas entre las distintas opciones, regularmente relacionadas con aspectos de escritura y uso de puntuaciones; y (iii) como consecuencia de lo anterior, la complejidad para migrar un manuscrito rechazado al buscar suministrarse para evaluación en otra revista científica, requiriéndose estructurar nuevamente el documento de forma completa.

A partir de los resultados observados, se proponen las siguientes estrategias: (i) influir en organismos reguladores de la ciencia en generar políticas públicas que estandaricen el formato de estilo editorial, con especificaciones generales y particulares de aplicación de cada área de conocimiento; (ii) elaborar programas educativos, significativos y eficientes de formación en estilos editoriales para estudiantes, profesores e investigadores; (iii) crear servicios institucionales en formato de centros de escritura o de soporte académico que influyan en la propiciación de niveles suficientes de alfabetización científica y propiciar la generación de publicaciones; y (iv) considerar, principalmente, dentro de los programas académicos de bibliotecología y ciencias de la 
información, la integración de perfiles profesionales o de especialidad sobre producción y comunicación científica.

Aunque existe evidencia de múltiples estudios sobre formación de investigadores en instituciones de educación superior, aún se carece de marcos teóricos que permitan orientar los análisis descriptivos de carácter nacional (Rivas Tovar, 2004). Regularmente las visiones de formación en investigación, ya sea que provienen de procesos de instrucción (especialmente a través de programas de doctorado) o aquellos basados en las políticas científicas gubernamentales que desarrolla cada país, de lo cual resultan propuestas a nivel macrosistema, que no necesariamente abarcan cuestiones particulares, como sucede con los aspectos normativos vinculados a la edición de documentos científicos.

Según el planteamiento anterior, la mayoría de las propuestas se centran en alternativas para el desarrollo de condiciones institucionales, pero dejan de lado los aspectos personales de los profesores que ingresan al ambiente de la investigación, los cuales pueden ser de carácter familiar, económico, laboral o de formación previa (Moreno Bayardo 2011). Esta última es la que comprende el uso y adaptación a condiciones de manuales de estilo, en donde, no ha sucedido ningún proceso formativo que desarrolle capacidades de conocimiento o de habilidades de adaptación editorial.

En los últimos años, se ha puesto de manifiesto el deseo de países latinoamericanos (especialmente México) de reducir la dependencia de conocimiento proveniente de economías desarrolladas. Por tanto, ha promovido de forma insistente que los profesores universitarios desarrollen actividades de investigación y estas se manifiesten en publicaciones, promoviendo sistemas editoriales de revistas científicas de calidad. Para Figueroa Delgado (2009), estas condiciones no se logran como un proceso natural de evolución, sino que requieren la adquisición de capacidades sistemáticas de profesores e investigadores para formar una infraestructura científico-tecnológica, cuya base sea la generación de conocimiento propio.

La situación en orientación de políticas de ciencia y tecnología en México, observa que el problema no han variado en el tiempo, debido al predominio y poder ejercidos por las élites científicas, quienes, en su afán de lograr un 
progreso científico, han dejado de lado elementos básicos en la definición de políticas específicas y formas de subsanar condiciones básicas del proceso de investigación (Casas Guerrero 2004). De acuerdo con la Organización de Estados Iberoamericanos para la Educación, la Ciencia y la Cultura (OEI 2012), el conocimiento científico es una de las principales riquezas de la sociedad contemporánea, necesario para impulsar el desarrollo económico y social, con lo cual, se acuñan términos como 'economía del conocimiento', en donde todos los países ajustan sus modelos a la medida de sus posibilidades, siendo un ejemplo de ello, la definición de sistemas editoriales vinculados a la comunicación de la ciencia.

Las tendencias actuales en los modelos de edición y comunicación científica experimentadas por las comunidades investigadoras se centran en lo siguiente: ineficacia para prevenir y detectar fraudes o la re-publicación de mismos contenidos; inconsistencia y reducida fiabilidad en las revisiones, que se traduce en valoraciones contradictorias de un mismo manuscrito; el carácter elitista determinado por la posición y prestigio de las personas e instituciones; y la poca consideración de ideas innovadoras, regularmente contrarias a las teorías dominantes (Fresco Santalla, 2013). Aunado a todo ello, debe considerarse la presión por publicar y el número mayor de documentos aspirantes a ser evaluados para publicación, al grado en que las imprecisiones y variabilidad en los formatos editoriales provocan procesos de sobreinformación y, en consecuencia, la frecuente desestimación de documentos viables a la publicación tomando sólo como referencia elementos de formato y no de contenido.

Es cierto que la profundidad y el contenido de los artículos científicos varían conforme avanza el conocimiento, lo que permite la reproductividad adecuada de lo expuesto por los investigadores, considerándose que hasta ahora, el medio más reconocido para comunicar conocimiento se basa en el artículo científico (López Borrul, 2017). Estas cuestiones no son un problema en los procesos de comunicación del conocimiento, no obstante, la situación se complica cuando los aspectos relacionados con el formato editorial superan al propio contenido que se desea publicar y no existen sistemas editoriales nacionales suficientemente eficaces para facilitar los procesos de publicación. Para García Pérez y García Aretio (2014), la promoción de la investigación en profesores universitarios es un proceso inconcluso si no se llega a la 
publicación con el asentimiento de la comunidad científica. Por lo tanto, se prioriza la existencia de sistemas editoriales estandarizados.

\section{Bibliografía}

ALONSO-GAMBOA, J.O., F.R. REYNA ESPINOSA y L.A. SANCHEZ-ISLAS, 2015. Características y calidad editorial de las revistas científicas mexicanas: la aportación de Latindex. Ciência da Informação, 44(2), 276-289. ISSN 15188353.

ASENCIO-CABOT, E. y H. IBARRA LÓPEZ, 2018. Experiencia en la preparación de investigadores como autores y revisores de artículos científicos. Biblios, 70, 485-459. ISSN 1562-4730. doi: 10.5195/biblios.2018

BORNMANN, L., 2019. Does the normalized citation impact of universities profit from certain properties of their published documents - such as the number of authors and the impact factor of the publishing journals? A multilevel modeling approach. Journal of Informetrics, 13, 170-184. ISSN: 1751-1577. Doi: 10.1016/j.joi.2018.12.007

CASAS GUERRERO, R., 2004. Ciencia, tecnología y poder: elites y campos de lucha por el control de las políticas. Convergencia: Revista de Ciencias Sociales, 11(35), 79-105. ISSN: 1405-1435

CONSEJO NACIONAL DE CIENCIA Y TECNOLOGÍA - CONACYT, 2018. Sistema de Clasificación de Revistas Mexicanas de Ciencia y Tecnología del Consejo Nacional de Ciencia y Tecnología. [Consulta: 1 junio 2019]. Disponible en: wmw. revistascytconacyt. $m x$.

CONSEJO NACIONAL DE CIENCLA Y TECNOLOGÍA - CONACYT, 2019a. Manual del Sistema de Clasificación de Revistas Mexicanas de Ciencia y Tecnología CMRCYT. Ciudad de México: CONACYT; SCIMAGO.

CONSEJO NACIONAL DE CIENCLA Y TECNOLOGÍA - CONACYT, 20196. Criterios SNI. [Consulta: 31 diciembre 2019]. Disponible en: https://www.conacyt.gob.mx/index.php/sni/convocatoriasconacyt/convocatorias-sistema-nacio $\% 20$ nal-de-investigadores-sni/marcolegal-sni/criterios-sni

CORDERO-ARROYO, G. et al., 2009. Publicaciones científicas y evaluación docente en México: un diagnóstico e intervención de profesores de educación y humanidades en una universidad estatal. Tiempo de Educar, 10(19), 149-168. ISSN: 1665-0824.

DE FILIPPO, D., S. MARUGÁN y E. SANZ-CASADO, 2014. Perfil de colaboración científica del sistema español de educación superior. Análisis de las publicaciones en Web of Science (2002-2011). Revista Española de Documentación Cientifica, 37(4): e067. ISSN: 0210-0614. doi: http://dx.doi.org/10.3989/redc.2014.4.1155 
EGUARAS, M., 2014. Manual de estilo: ¿por qué estandarizar y armonizar criterios? [Consulta: 1 junio 2019]. Disponible en: https:// marianaeguaras.com/manual-de-estilo-por-que-estandarizar-yarmonizar-criterios/

FIERRO, A., 2004. Contra el formato editorial. Psicothema, 16(2), 309-316. ISSN $0214-9915$.

FIGUEROA DELGADO, S.A., 2009. El papel del Estado en el avance de la ciencia y la tecnología: insumo vital de la construcción del desarrollo. En: S.A. Figuero Delgado, G. Sánchez Daza y A. Vidales Carmona. La ciencia y la tecnología en el desarrollo: una visión desde América Latina. Zacatecas, México: Universidad de Zacatecas, pp. 9-16. ISBN 978-607-7678-24-3.

FONDÓN, I., M.J. MADERO y A. SARMIENTO, 2010. Principales problemas de los profesores principiantes en la enseñanza universitaria. Formación Universitaria, 3(2), 21-28. ISSN: 0718-5006

FRESCO SANTALLA, A.M., 2013. Edición y comunicación cientifica: evolución y tendencias actuales. Tesis (Máster en Bibliotecas y Servicios de Información Digital, Universidad Carlos III de Madrid, España).

GANGA-CONTRERAS, F., L. PAREDES-BUZETA y L. PEDRAJA-REJAS, 2015. Importancia de las publicaciones académicas: algunos problemas y recomendaciones a tener en cuenta. IDESLA, 33(4), 111-119. ISSN: 07183429. DOI: http://dx.doi.org/10.4067/S0718-34292015000400014

GARCÍA PÉREZ, M. y L. GARCÍA ARETIO, 2014. Líneas de investigación y tendencias de la educación a distancia en América Latina a través de las tesis doctorales. REID. Revista Iberoamericana de Educación a Distancia, 17(1), 201230. ISSN: $1138-2783$

GAVIDIA, V., 2013. Los retos de la divulgación y enseñanza científica en el próximo futuro. Didáctica de las Ciencias Experimentales y Sociales, 19, 91-102. ISSN: 2255-3835.

KRATOCHVÍL, J., 2017. Comparison of the accuracy of bibliographical references generated for media citation styles by EndNote, Mendeley, RefWorks and Zotero. The Journal of Academic Librarianship, 43, 57-66. ISSN: 0099-1333.

LÓPEZ-BORRULL, A., 2017. Cambios y tendencias en la publicación de revistas científicas. En: Abadal, E. (Coord.). Revistas científicas: situación actual y retos de futuro. Barcelona, España: Universitat de Barcelona, pp. 221-237. ISBN: 978-849168-038-3.

LÓPEZ-VALDEZ, M., 2009. Guía de estilo editorial para obras académicas. México, D.F.: Ediciones del Ermitaño; Universidad Nacional Autónoma de México, Centro Regional de Investigaciones Multidisciplinarias. ISBN 978-607-764007-3.

MORENO BAYARDO, M.G., 2011. La formación de investigadores como elemento para la consolidación de la investigación universitaria. Revista de la Educación Superior, 158(2), 59-78. ISSN: 0185-2760. 
ORGANIZACIÓN DE ESTADOS IBEROAMERICANOS PARA LA EDUCACIÓN, LA CIENCIA Y LA CULTURA (OEI), 2012. Ciencia, tecnología e innovación para el desarrollo y la cohesión social. Programa iberoamericano en la década de los bicentenarios. Madrid: España. ISBN: 978-84-7666-240-3.

ORTOLL, E. et al., 2014. Principales parámetros para el estudio de la colaboración científica en big science. Revista Española de Documentación Cientifica, 37(4): e069. ISSN: 0210-0614. doi: http://dx.doi.org/10.3989/redc.2014.4.1142

PEI-SHAN, C., 2019. The Field-Specific Reference Patterns of Periodic and Non serial Publications. Journal of the Association for Information Science and Technology, 70(3), 283-292. ISSN:2330-1643.

PEINADO-RODRÍGUEZ, M., 2013. El papel de los medios de comunicación y la formación del pensamiento crítico. En: J.J. Díaz Manjarrez, A. Santiesteban Fernández y A. Cascajero Garcés. Medios de Comunicación y Pensamiento Crítico: nuevas formas de interacción social. Alcalá de Henares, España: Universidad de Alcalá de Henares, pp. 51-66. ISBN: 978-84-15834-22-9.

PÉREZ-ANAYA, O., 2017. Índice de Osk: una nueva medición bibliométrica para las revistas científicas. Revista Española de Documentación Científica, 40(2): e174. ISSN: 0210-0614. doi: http://dx.doi.org/10.3989/redc.2017.2.1418

PÉREZ-MORALES, R.W. y F.A. RODRÍGUEZ MANRIQUE, 2012. Formación de profesores de química a partir de la explicación de fenómenos cotidianos: una propuesta con resultados. Revista Eureka sobre Enseñanza y Divulgación de las Ciencias, 9(1), 124-142. ISSN: 1697-011X.

PRIETO-DÍAZ, V., et al., 2011. Impacto de las tecnologías de la información y las comunicaciones en la educación y nuevos paradigmas del enfoque educativo. Educación Médica Superior, 25(1), 95-102. ISSN: 0864-2141.

RIVAS TOVAR, L.A., 2004. La formación de investigadores en México. Perfiles Latinoamericanos, 25, 89-113. ISSN: 0188-7653.

RODRÍGUEZ-MENÉNDEZ, M., Z. GONZÁLEZ-CANTALAPIEDRA y M. GONZÁLEZ-POLO, 2016. Problemas frecuentes en la redacción de artículos científicos. EduSol, 16(57), 137-147. ISSN: 1729-8091.

SANCHO, J.M., 2010. Del sentido de la investigación educativa y la dificultad de que se considere para guiar las políticas y las prácticas. REICE: Revista Electrónica Iberoamericana sobre Calidad, Eficacia y Cambio en Educación, 8(2), 3446. ISSN: 1696-4713.

SENZ, S., 2014. Las guías de estilo editorial: historia, tipología, materializaciones y contenido. [Consulta: 1 septiembre 2019]. Disponible en: http://www.rededitores.sld.cu/las-guias-de-estilo-editorial-historia-tipologiamaterializaciones-y-contenido

VÉLEZ-CUARTAS, G. et al., 2014. Diversidad y reconocimiento de la producción académica en los sistemas de evaluación de la investigación en Colombia. Revista Española de Documentación Cientifica, 37(3): e056. ISSN: 0210-0614. doi: http://dx.doi.org/10.3989/redc.2014.3.1133 
VILCHES, A., J. SOLBES y D. GIL, 2004. ¿Alfabetización científica para todos contra ciencia para futuros científicos? Alambique, 41, 89-98. ISSN: 11339837. 